\title{
Molecular and Serological Characterization of Iris Yellow Spot Virus, a New and Distinct Tospovirus Species
}

\author{
I. Cortês, I. C. Livieratos, A. Derks, D. Peters, and R. Kormelink
}

First, second, fourth, and fifth authors: Department of Virology, Wageningen Agricultural University, the Netherlands; and third author: Bulb Research Centre, Lisse, the Netherlands.

Current address of I. Cortês: Secção de Protecção de Plantas, UTAD, Vila Real, Portugal.

Current address of I. C. Livieratos: Imperial College of Science, Technology \& Medicine, London, England.

Accepted for publication 27 August 1998.

\section{ABSTRACT}

Cortês, I., Livieratos, I. C., Derks, A., Peters, D., and Kormelink, R. 1998. Molecular and serological characterization of iris yellow spot virus, a new and distinct tospovirus species. Phytopathology 88:1276-1282.

A new tospovirus was identified in iris cultivations in the Netherlands. Both serological comparisons and sequence determination of the S RNA demonstrate that this virus represents a new and distinct species, belonging to a separate serogroup, and for which the name iris yellow spot virus
(IYSV) is proposed. The disease symptoms on iris are characterized by yellow spots on the leaves. Its experimental host range is very narrow and, in addition to iris, only includes Nicotiana benthamiana and Datura stramonium. The nucleoprotein of IYSV shows only 30 to $44 \%$ sequence identity with those of other tospoviruses identified so far; the highest homology being found with the tospovirus species of serogroup IV.

Additional keywords: Bunyaviridae, INSV, phylogenetic tree, TSWV.
Based on molecular studies, tomato spotted wilt virus (TSWV) was the first phytopathogenic virus identified as a member of the family Bunyaviridae, a large family of mostly arthropod-borne enveloped RNA viruses, originally thought to be restricted to animals (11). During the past 10 years, due to intensified studies and the development of facile molecular technologies, it has become clear that TSWV is not the sole plant-infecting bunyavirus. In 1990 a second virus, impatiens necrotic spot virus (INSV), was found in the United States (21) and later also in Italy and the Netherlands $(3,31)$. This virus is mainly restricted to ornamental plant species and is serologically distinct from TSWV, its nucleoprotein showing only 55\% amino acid sequence identity with that of TSWV $(21,22)$. Further studies by De Ávila et al. (7) demonstrated the existence of two more related species, tomato chlorotic spot virus (TCSV) and groundnut ringspot virus (GRSV). In 1995, these viruses were taxonomically placed into a separate genus within the family Bunyaviridae, the genus Tospovirus (12). Since then, even more tospovirus species have been identified and distinguished based on nucleoprotein $(\mathrm{N})$ serology, $\mathrm{N}$-protein sequences, and vector specificity.

Using polyclonal and monoclonal antisera directed to the viral nucleoproteins, recognized tospoviruses have been placed into four distinct serogroups so far. TSWV is the representative of serogroup I (5), TCSV and GRSV are placed in serogroup II (4), INSV in serogroup III $(21,22)$, and watermelon silver mottle virus (WSMV) $(16,32,33)$ and peanut bud necrosis virus (PBNV) $(13,25,27)$ in serogroup IV.

Since 1992, disease symptoms have been occasionally encountered in cultivations of Iris hollandica Tub. in the Netherlands. The symptoms on naturally infected iris plants consisted of typical chlorotic spots that, in a progressed stage of the disease, developed into yellow and necrotic spots. Depending on the iris cultivar, the

Corresponding author: R. Kormelink

E-mail address: richard.kormelink@medew.viro.wau.nl

Publication no. P-1998-1014-02R

(C) 1998 The American Phytopathological Society percentage of infected plants ranged between 50 and 90\%. Often, thrips were observed within the iris cultivations, and chemical spraying against these resulted in the absence of diseased plants, hence suggesting that the disease was being caused by a tospovirus. Electronmicroscopic analysis of tissue from diseased iris indeed confirmed the presence of spherical particles, 80 to $120 \mathrm{~nm}$ in diameter (data not shown), characteristic for tospoviruses (30).

Here, we describe this new tospovirus isolated from I. hollandica cultivations in the Netherlands, for which the name iris yellow spot virus (IYSV) has been coined. Serological and nucleotide sequence data presented in this paper demonstrate that this virus is a distinct and new tospovirus species, belonging to none of the described serogroups.

\section{MATERIALS AND METHODS}

Viruses and plants. IYSV was isolated from I. hollandica during the cultivation season in 1992 and maintained in Nicotiana benthamiana Domin. by mechanical inoculation. Tospovirus isolates TSWV BR-01 (6), TCSV BR-03 (6), GRSV SA-05 (6), INSV NL-07 (3), and WSMV Tospo-to (16), used in serological studies, were also maintained on $N$. benthamiana.

For the establishment of the host range, leaf tissue of IYSV-infected $N$. benthamiana was ground in $0.01 \mathrm{M}$ sodium sulfate buffer, $\mathrm{pH} 7.0$, containing $0.1 \%$ sodium sulfite, and mechanically inoculated on N. benthamiana, N. glutinosa, N. rustica, N. clevelandii, Gomphrena globosa, Chenopodium quinoa, Chenopodium amaranticolor, Emilia sonchifolia, Cucurbita sativus, Vigna unguiculata, Phaseolus vulgaris, Pisum sativum, Datura stramonium, Lycopersicum esculentum, Petunia hybrida, and Impatiens spp. The plants were kept under greenhouse conditions and assayed for symptom expression.

Thrips transmission. Populations of 1-day-old Thrips tabaci and Frankliniella occidentalis larvae were placed on infected leek plants for 2 days, and then transferred in groups of approximately 10 larvae to petunia leaves for two periods of 2 days each. The larvae pupated in the last period and were again tested for their infectivity after emergence to adults in one inoculation access period. 
Virus purification. Nucleocapsids of IYSV were purified from systemically infected $N$. benthamiana as described by De Ávila et al. (7), but subsequently applied on a 25 to $45 \%$ cesium sulfate gradient for further purification. For sodium dodecyl sulfate polyacrylamide gel electrophoresis (SDS-PAGE) analyses (20) of the N proteins, nucleocapsids of TSWV, INSV, TCSV, GRSV, and WSMV were likewise purified.

Purified nucleocapsid material of IYSV was used to produce a polyclonal antiserum to the $\mathrm{N}$ protein. Rabbits were intradermally injected two times, at a 2-week interval, with an emulsion of incomplete Freund's adjuvant and purified nucleocapsids (1:1, wt/vol). Blood was collected 2 weeks after the last injection.

Viral (v) RNA of IYSV was extracted from either a semipurified preparation obtained after centrifugation through a sucrose cushion or from purified nucleocapsids obtained after $\mathrm{CsSO}_{4}$ gradient centrifugation (3). The RNA was isolated by treatment of nucleocapsids with $1 \%$ SDS followed by phenol extraction and ethanol precipitation.

Serological analyses. IYSV was serologically compared with other tospovirus species by double-antibody sandwich-enzyme-linked immunosorbent assay (DAS-ELISA) (2) using polyclonal antisera directed against the N protein of each virus. The antisera for TSWV, INSV, TCSV, and GRSV were previously prepared by De Ávila et al. (6). Polyclonal anti-N serum for Tospo-to was kindly supplied by G. Adam (University of Hamburg).

Molecular cloning and sequence determination. To obtain IYSV $S$ RNA-specific cDNA clones, two different methodologies were followed: (i) construction of a cDNA library and (ii) reverse transcriptase polymerase chain reaction (RT-PCR). In both cases, firststrand cDNA was primed with J1 (5'-dTTTTTTCTAGAGCAATC$3^{\prime}$ ), complementary to the first eight terminal nucleotides of the $3^{\prime}$ end conserved for all known tospoviruses. Second-strand synthesis was performed according to Gubler and Hoffman (15). Doublestranded cDNA was made blunt-ended using T4 DNA polymerase and subsequently cloned into pUC119 after the addition of BamHI linkers (24).

In the second methodology, first-strand cDNA was amplified using primers J1 and J2 (dATAGTTAAGCATAAACACAA) complementary to nucleotide position 2,585 to 2,603 of WSMV S RNA (numbered from the $5^{\prime}$ end of the vRNA) using the Expand Long Template PCR System (Boehringer Mannheim GmbH, Mannheim, Germany). Fragments obtained after PCR, and specific for IYSV $\mathrm{S}$ RNA, were blunt-end cloned into the EcoRV site of pSK+.

DNA sequencing was performed by the dideoxynucleotide chain termination method (26) on an ABI Automatic Sequence machine (Applied Biosystems, Inc., Foster City, CA). Nucleotide and amino acid sequences were compiled and analyzed using the GCG package of the University of Wisconsin (10).

Expression of the $\mathbf{N}$ gene in Escherichia coli. For convenient cloning of the IYSV S RNA viral complementary (vc)-open reading frame (ORF), the putative $\mathrm{N}$ gene, the ORF was PCR-amplified using oligonucleotides P1 (dCCCGAGGATCCATGGCTACCGTTAGGG), complementary to nucleotides 3,020 to 3,037 of the vRNA strand, and P2 (dCCCGAGGATCCAAATTAATTATATCTATCTTTCTTGG), identical to nucleotides 2,211 to 2,236 of the vRNA strand. After PCR amplification, the DNA was digested with $N c o$ I and BamHI and subsequently cloned in the E. coli expression vector $\mathrm{pET}-11 \mathrm{t}$, resulting in plasmid $\mathrm{pET}-11 \mathrm{t} / \mathrm{N}$. Due to the use of oligonucleotide P1, containing an altered IYSV sequence for the introduction of an $\mathrm{NcoI}$ cloning site, the second amino acid at the $\mathrm{N}$-terminus of the putative $\mathrm{N}$ protein was changed from serine into alanine.

For analysis on the $\mathrm{N}$ gene expression, E. coli BL21 cells were transformed with the pET-11t/N construct and subsequently induced with isopropyl- $\beta$-D-thiogalactopyranoside (IPTG) as described by Kormelink et al. (19). Proteins synthesized were analyzed on a $15 \%$ SDS polyacrylamide gel (20), followed by western immunoblot analysis on poly-vinylidene-difluoride (PVDF) membrane, using polyclonal anti-N serum.

\section{RESULTS}

Host range and symptoms. Some plant species previously utilized to determine the host range of different tospoviruses (6) as well as for maintenance of the virus were inoculated with IYSV. Whereas most plant species reacted locally with chlorotic, pin point, or necrotic lesions, IYSV only systemically infected $N$. benthamiana and $D$. stramonium. $N$. benthamiana responded with local lesions within 8 days and with chlorotic spots and leaf deformation on younger leaves after 12 days postinoculation. Local symptoms on $D$. stramonium resembled those on $N$. benthamiana, but systemically a mottling was observed. Petunia hybrida reacted with symptoms characteristic for tospoviruses (i.e., with local necrotic lesions only). No local or systemic symptoms could be observed on Phaseolus vulgaris, Pisum sativum, L. esculentum, $N$. glutinosa, or $N$. rustica, all of which appeared not to be susceptible to IYSV. When I. hollandica plants were inoculated with IYSV, they developed the symptoms again as observed on the plants collected from the field in 1992 (Fig. 1).

Thrips transmission. Numerous local lesions were observed on the petunia leaves that were infected by the T. tabaci larvae in the second inoculation access period and the adults after emergence. Our results support the observation made in Israel that $T$. tabaci can acquire IYSV from infected leek plants as shown in ELISA using polyclonal anti-IYSV serum (A. Gera, personal communication). No transmission by $F$. occidentalis was observed. Studies on the role of other thrips species as vectors of IYSV are ongoing.

Serological differentiation of IYSV with other tospoviruses. To determine the serological relation of IYSV with other established species within the genus Tospovirus, samples of IYSV-infected $N$. benthamiana were compared with TSWV, GRSV, TCSV,

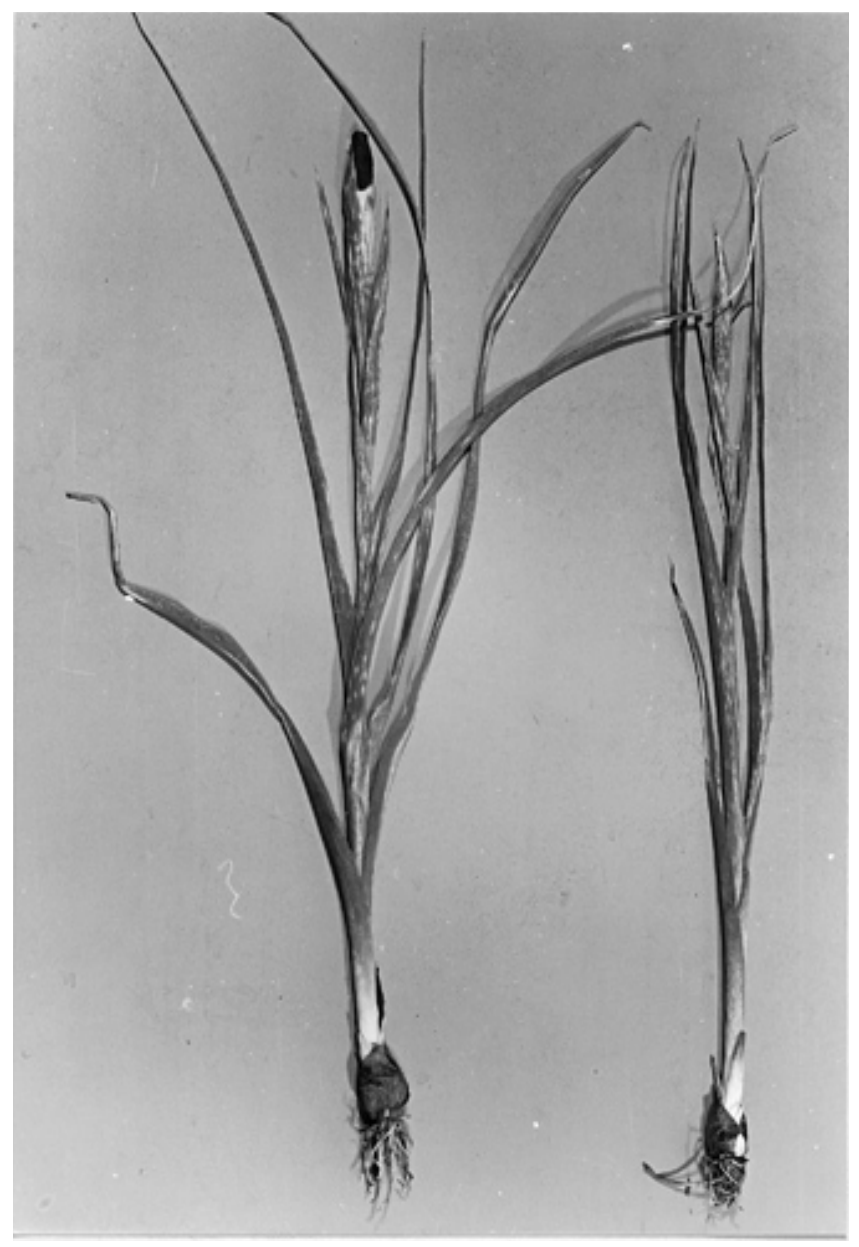

Fig. 1. Iris hollandica plant naturally infected with iris yellow spot virus. 
INSV, or WSMV in DAS-ELISA. The latter viruses compose the members of serogroups I to IV within the genus Tospovirus. Positive reactions were obtained with all homologous combinations (Fig. 2), and weak reactions were obtained in the heterologous combinations between TSWV, TCSV, and GRSV, as expected (5). None of the recognized tospoviruses reacted with the antiserum raised against the IYSV N protein, and none of the other polyclonal anti-N sera recognized the IYSV $\mathrm{N}$ protein. These results show that IYSV is serologically distinct from the other tospoviruses.

The size of IYSV nucleoprotein. To estimate the size of IYSV $\mathrm{N}$ protein and compare it with those of other tospoviral $\mathrm{N}$ proteins, nucleocapsids were purified from IYSV-infected $N$. benthamiana and applied to a $15 \%$ SDS polyacrylamide gel. The results (Fig. 3) revealed that the N protein of IYSV had an estimated $M_{\mathrm{r}}$ of $30 \mathrm{kDa}$. This protein is larger than the $\mathrm{N}$ proteins of TSWV (28.8 kDa), TCSV (28.7 kDa), GRSV (28.8 kDa), and INSV (28.7 kDa), but still smaller than that of WSMV $(30.6 \mathrm{kDa})$.

RNA analysis. vRNA of IYSV was purified from nucleocapsids obtained after $\mathrm{CsSO}_{4}$ gradients and resolved on a $1 \%$ agarose gel under denaturing conditions (1). After electrophoresis, the gel revealed the presence of three IYSV RNA bands that approximately corresponded in size to the $\mathrm{S}(2.9 \mathrm{~kb}), \mathrm{M}(4.8 \mathrm{~kb})$, and L RNA $(8.9 \mathrm{~kb})$ of TSWV (Fig. 4).

Molecular cloning and sequence determination of IYSV $S$ RNA. The first fragment specific for IYSV S RNA was obtained using oligonucleotide $\mathrm{J} 1$ to prime first-strand cDNA synthesis, followed by PCR amplification using J1 and J2. A DNA fragment of 962 bp, expected to include the N gene, was obtained (Fig. 5, P3). Another fragment (Fig. 5, P1) covered the full-length S RNA and was amplified using only primer J1, containing the eight conserved terminal nucleotides of tospoviral RNAs. After having confirmed the specificity of these fragments for IYSV S RNA on northern blots, they were purified and cloned into pSK+ or pUC119. The nucleotide sequence of P1 and P3 was determined and, based on these data, new primers were designed for the amplification of internal fragments of the IYSV S RNA. The position of the clones obtained and sequenced, as well as the primers used for internal sequencing or PCR amplification, are shown in Figure 5.

In addition to PCR amplification of IYSV S RNA-specific fragments, a cDNA library was made of IYSV. For this purpose, first- strand cDNA synthesis was primed with $\mathrm{J} 1$, followed by secondstrand synthesis and cloning in pUC119. The library obtained was screened with PCR fragment P3 (Fig. 5) and yielded one clone (C5) that was also sequenced to confirm the nucleotide sequencing results obtained from PCR-derived clones. Using the methodologies of PCR amplification and the screening of a cDNA library, a set of overlapping clones to IYSV S RNA was obtained that yielded a restriction map covering approximately 3,000 nucleotides (Fig. 5). Each stretch of the S RNA was sequenced at least twice in both orientations, using clones obtained from at least two independent cDNA synthesis and PCR amplification reactions.

Characteristics and predicted gene products of IYSV S RNA. The IYSV S RNA segment is 3,105 nucleotides (Fig. 6) and shows features typical for tospoviral RNAs: (i) the first eight nucleotides of terminal sequences are identical to the 5' and 3' termini of all known tospoviral RNA segments; (ii) the RNA has complementary termini that are involved in the formation of a stable panhandle structure; and (iii) like the S RNA segments of all known tospoviruses, the IYSV S RNA contains two ORFs in an

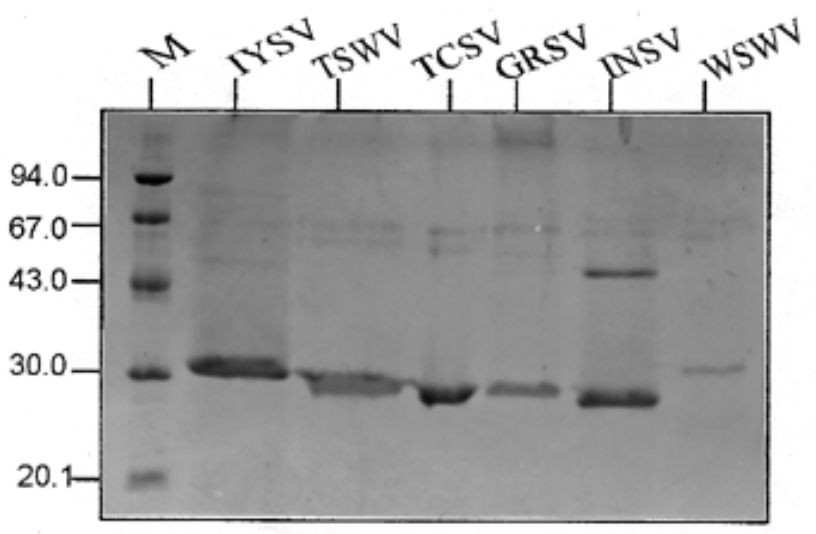

Fig. 3. Comparison of the nucleocapsid proteins of tospoviruses from different serogroups. Proteins were resolved on a $15 \%$ sodium dodecyl sulfate polyacrylamide gel and stained with Coomassie brilliant blue. Low molecular weight (LMW) size markers (Amersham Pharmacia Biotech, Uppsala, Sweden) are included on the left side of the gel.

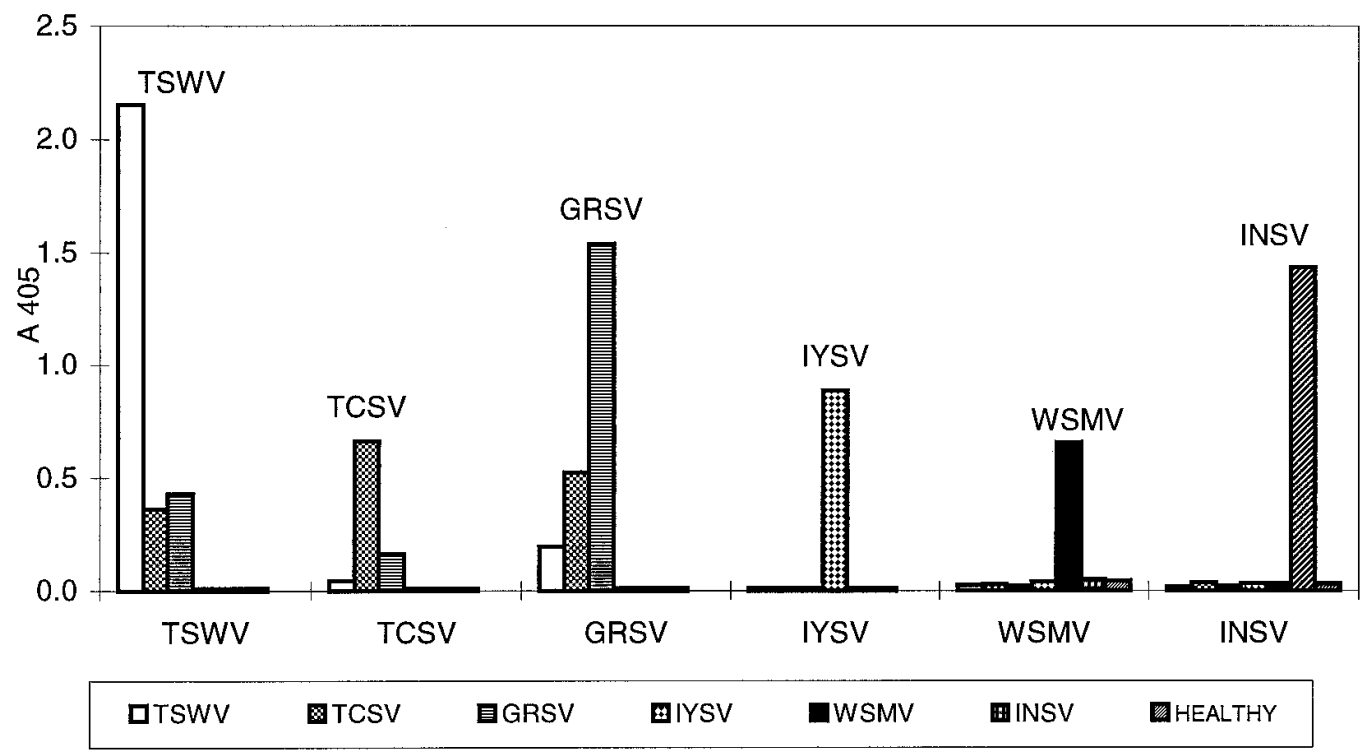

\section{Nucleocapsid protein antisera}

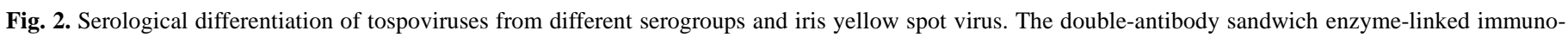
sorbent assay was performed using polyclonal antisera directed against their respective N proteins and the extracts from infected plants as antigen source. 
ambisense gene arrangement. Both v- and vc-sense ORFs are preceded by a noncoding leader sequence of 70 nucleotides, with an exact inverted complementarity at the ends for $15 \mathrm{nu}-$ cleotides. An internal noncoding region consisting of $810 \mathrm{nu}-$ cleotides is located between nucleotide position 1,400 and 2,216. It contains an inverted sequence of A-rich stretches followed by U-rich stretches that can be folded into a hairpin structure. A sequence conserved among the top of the hairpins in the $\mathrm{M}$ and S RNAs of TSWV and INSV $(8,9,17,23)$ was also found at the

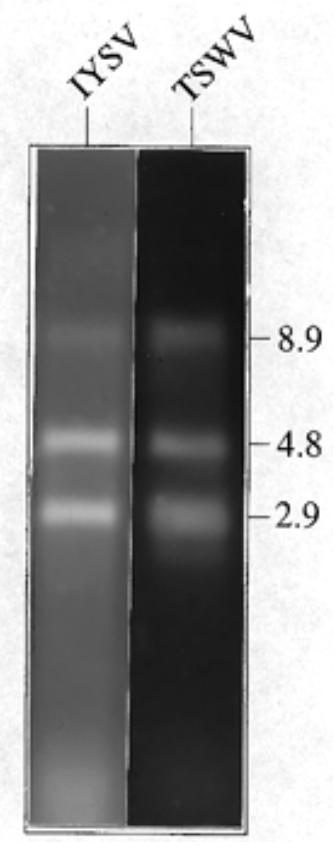

Fig. 4. Analysis of purified nucleocapsid RNA of iris yellow spot virus on a denaturing $1 \%$ agarose gel. As control, tomato spotted wilt virus (TSWV) nucleocapsid RNA was included. The sizes of the TSWV RNA segments are indicated on the right side of the gel in kilobases. top of the hairpin (CAAACUUUGG) of IYSV S RNA, suggesting that this nucleotide sequence may have an important function in the termination of transcription of ambisense RNA ORFs $(9,17)$.

The ORF found in v-sense, starting with a AUG codon at position 71 and terminating with a UGA codon at position 1,400, encodes a protein with a predicted molecular weight of $50.1 \mathrm{kDa}$. By analogy to known tospoviruses, this protein is considered to be the $\mathrm{NS}_{\mathrm{S}}$ protein. The second ORF, in vc-sense, starts with an AUG codon at position 3,035 and terminates with a UAA codon at position 2,216 (numbered from the $5^{\prime}$ end of the vRNA). This ORF encodes the putative $\mathrm{N}$ protein with a $M_{\mathrm{r}}$ of $30.5 \mathrm{kDa}$.

Expression of the vcORF of IYSV S RNA. To demonstrate that the vcORF indeed represents the $\mathrm{N}$-protein gene, this sequence was expressed in E. coli, using the expression vector pET-11t. To this end, the vcORF was PCR-amplified using primers P1 and P2 and subsequently cloned as a NcoI-BamHI fragment in pET-11t. Clones containing the viral gene were selected and induced for protein expression. The protein synthesized had the expected size of the IYSV N protein and reacted with IYSV polyclonal anti-N serum (Fig. 7). This result confirmed that the IYSV vcORF indeed represents the $\mathrm{N}$-protein gene.

\section{DISCUSSION}

Thus far, complete or partial sequence information of the S RNA of 6 out of the 10 established (14) tospovirus species were available: TSWV (9), TCSV (4), GRSV (4), INSV (8,22), WSMV (16, 32 ), and PBNV(27). In this paper, we present such data, as well as some biological data, of a new tospovirus isolated from iris cultivations in the Netherlands. Data on thrips transmission, serology, host range, and the S RNA sequence demonstrate unequivocally that it represents a new tospovirus for which the name iris yellow spot virus (IYSV) is proposed. This brings the total of distinct species within the genus Tospovirus to 11 .

Based on the S RNA sequence data and the described N-protein sequence data, a phylogenetic tree reflecting the evolutionary positions of the various tospoviruses could be made. The tree based

\begin{tabular}{rrrrrrr}
400 & 800 & 1200 & 1600 & 2000 & 2400 & 1 \\
\hline
\end{tabular}
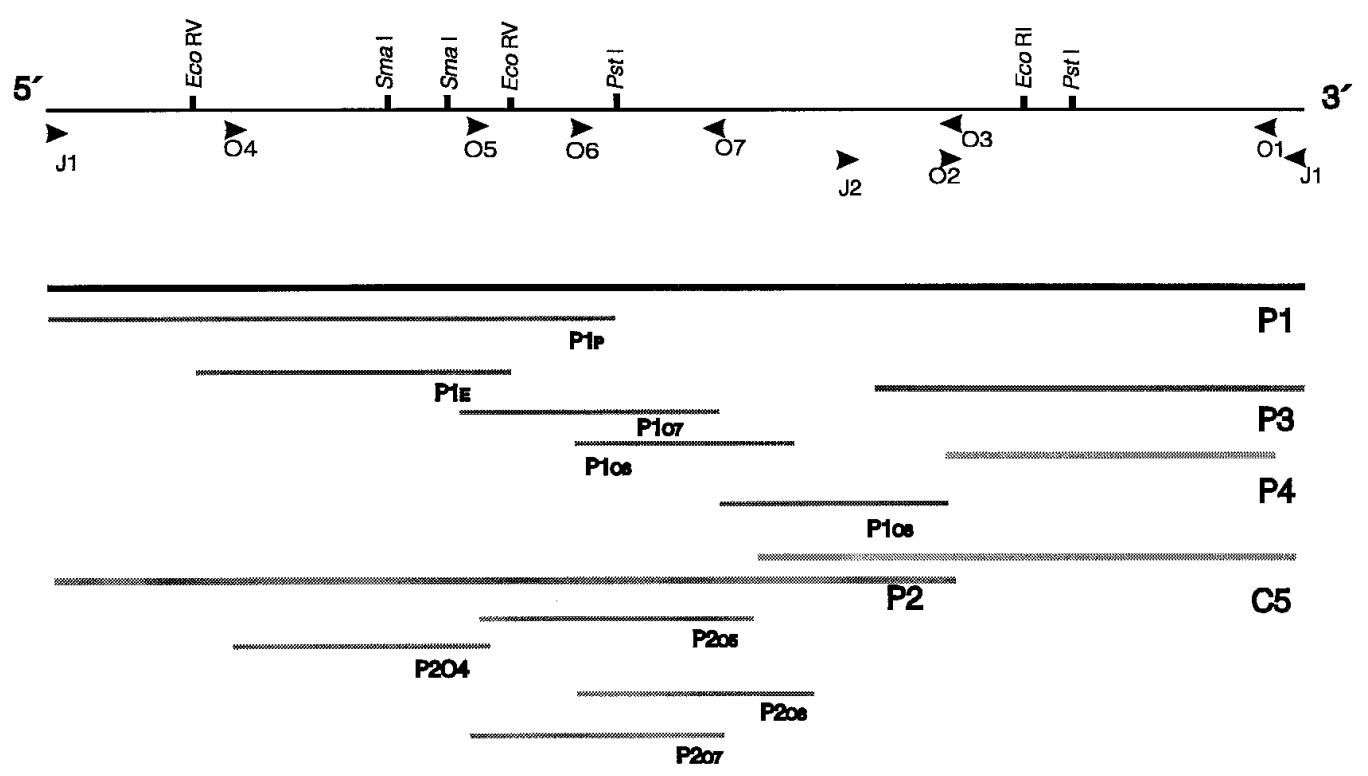

Fig. 5. Cloning strategy for the iris yellow spot virus S RNA. The arrowheads indicate the primers used for cDNA synthesis and polymerase chain reaction (PCR) amplification. The clones derived from PCR are indicated with a P and the cDNA clones with a $\mathrm{C}$. The larger clones were sequenced either by subcloning or by sequencing directly with primers. Subclones are referred to with the name of the original clone plus the designation of the enzyme utilized for digestion or primer used. 
on the N-protein sequence reveals, in agreement with earlier reported studies, a clustering of TSWV, TCSV, GRSV, and INSV in one branch and a clustering of IYSV with PBNV and WSMV (Fig. 8). Multiple sequence alignments (using PRETTY of the
GCG package) only showed short stretches of amino acids conserved for all tospoviral $\mathrm{N}$ genes.

Based on computer alignments, more similarities were found that supported the clustering of IYSV with the Asian isolates WSMV AGAGCAAUCGAGGUACAAAACAAAAUCAUCAGCAGCUUGAAAAAGAUAAUUAAGAAAUACUACUUCAGACAUGUCUACCGUUAGGACUACGGCAGUCGAUUUCCUUUCGAACUACGGAAUAUCUUGUGAC

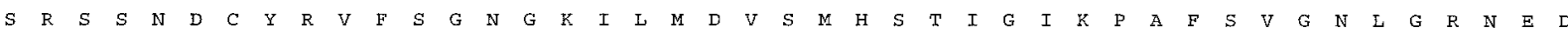
UCUCGUUCAAGCAACGAUUGUUACAGGGUAUUCUCUGGUAAUGGCAAAAUUUUAAUGGAUGUUUCCAUGCACUCAACGAUCGGAAUCAAACCUGCUUUCAGUGUUGGGUAAUCUUGGGCAGAAAUGAAGACA

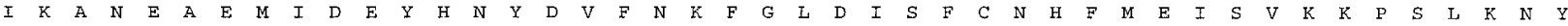
UUAAAGCUAAUGAGGCUGAGAUGAUAGAUGAAUAUCACAACUAUGAUGUUUUCAACAAAUUUGGUCUUGAUAUUAGUUUUUGCAACCACUUCAUGGAGAUAUCUGUAAAGAAACCUUCUCUAA.AGA.UUJA

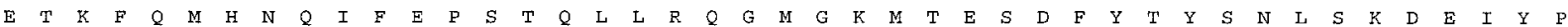
UGAGACAAAGUUUCAGAUGCACAAUCAGAUAUUUGAGCCUUCAACACAAUUGCUCAGGCAAGGUAUGGGCAAAAUGACUGAAUCUGAUUUCUAUACCUAUUCAAACCUUUCUAAAGACGAAAUAUACCCG

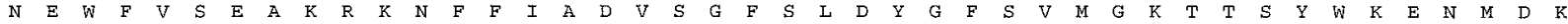
AACGAAUGGUUCGUAAGUGAAGCAAAGAGAAAAAACUUCUUCAUAGCUGAUGUUUCUGGAUUCAGCUUAGAUUAUGGAUUCUCUGUGAUGGGAAAAACUACUUCCUACUGGAAAGAGAAUAUGGACAAAA

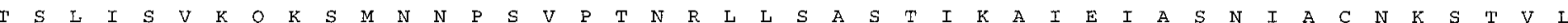
CAUCUCUAAUAUCAGUAAAGCAGAAAUCAAUGAAUAACCCUUCAGUUCCUACAAACAGACUCUUAUCAGCUUUCCACAAUAAAAGCAAUAGAAAUAGCAUCAAACAUUGCCUGCAAUAAAUCUACUGUUCU

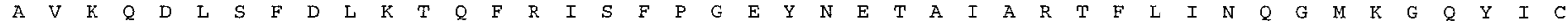
UGCAGUCAAACAAGAUUUGUCUUUUGACCUUAAGACUCAGUUCCGAAUCUCUUUCCCGGGAGAAUACA.AUGAAACAGCUAUAGCAAGAACCUUCUUAAUCAACCAAGGA.AUGAAGGGUCAAUAUAUAUGU

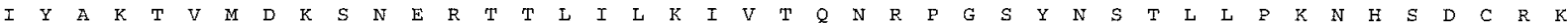
AUAUAUGCUAAAACAGUGAUGGAUAAAUCAAAUGAAAGGACAACGUUGAUUUUGAAAAUUGUCACUCAGAACAGGCCCGGGAGCUAUAACUCAACACUGCUUCCCA.AAAAUCAUUCAGACUGUAGGAAAG

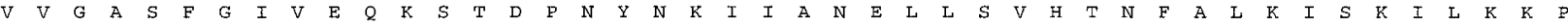
UGGUCGGAGCCAGUUUUGGUAUUGUCGAACAAAAAUCA.ACUGAUCCGA.AUJACAACAAAAUAAUUGCA.AAUGA.AUUAUUGUCUGUGCACACAAAUUUUGCUUUUAAAGAUAUCAAAGAUCCUGAAAAAACC

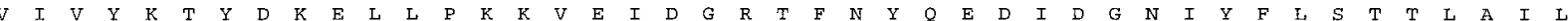
UGUGAUAGUUUAUAAAACUUAUGAUAAGGAAUUGUUGCCAAAAAAAGUGGAGAUAGACGGUAGGACAUUUAAUUAUCAAGAAGACAUCGAUGGAAAUAUUUACUUCUUAUCCACCACUCUUGCAAUCCUA

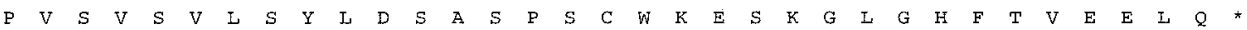

CCUGUGUCAGUCUCAGUCUUGUCAUAUCUUGACUCUGCUUCUCCAUCCUGCUGGAAAGAGUCCAAAGGUUUAGGACACUUCACUGUAGAAGAGCUGCAGUGAUUGGAUUAGUUAAUUCUGUCGUUUUAAUU

UAAGUUUGCUUAUUUUUAUCGUUUAUCAUUUAAGUUUUAAUAUUAUAUAAAUUUAUUCUAUUGUGUGUGUUAAGUUUGUUUGCAUUGUUUAAAAUAUCAUUUACUCAUUUAAUCUGUAUUAUAAAUAAAA

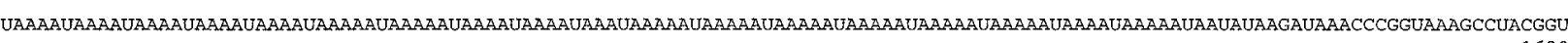

CAAAUUCUAAGUUCUAUCUAAGACUUAGAGUUUGACUGUAGGUUUUAUUGGCUUUUGGAUUAGUUUAUUUUAAUAUGUUGUCUUUGUGUUAUUUUAAAAUUGCUUUUUCUCUUAAUUUAAGUUUGUUAUU

UCAUUCCACCAGAAAGUAUAAUAUAAAUAAAUCAAACAUUCGUCGUUUAAAUUUGAAAUAUAUAUAAUGAGAGCUUAAAGGCAAAUUUUGGCCUAAAUGAUUAGAUUACUCCAGCCaAAGUUUGCCCGUA 1950

GGCUUUUUGGUUUUUGGUUUUUAAUUUUUUAUUUGGUUUUUAUUUUGUUUAUUUAACAUAAUUAAAAACUCUUAAACACAUUUAACAAGCACUUAAAAUUAUACUGAAAUAGURAAACUUAACACAC 2080 AAUUAAAUUAUAUAAACACACAAGAAGAAGAUUACAAAUAAGACUUAAACAGAUUUAAACAUUAAACACAARAUGACUGGAAACACUUAUAAAACUUAACUAACACAAAUACUGAUUAAUAAUAUAUAAU 2210 AAAUUAAUUAUAUCUAUCUUUCUUGGAGGGAGUUUUGGGUUUAGAAGACUCACCAAUGUCUUCAACAAUCUUUCCAAAAGCACCUUCAAGAAUUUUAAUCUGUUCAUUGAAUUUGAUCAAUGAAGCAGCA

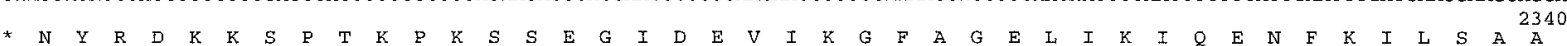
CCCGAAGUUCCAGGAGUGCAGUCAGUGAGGAUCUUGAUAGUUUCAUCAAAAAUCUUUUUGUAUUUCUCUGUGAAUUUGAAUUCCUUUGCUGCCAUGACUCUUGCAACUUUGCAGAGUUGCUCGUAAGUUG $\begin{array}{lllllllllllllllllllllllllllllllllllllllllllllllllll}G & S & T & G & P & T & C & D & T & \text { L } & I & K & I & T & E & D & F & I & K & K & Y & K & E & T & F & K & F & E & K & A & A & M & V & R & A & V & K & C & L & \& & E & Y & T & S\end{array}$ AGAAUCUGCUUAUACCGAGUGCUUCCUUUUUUGACAUUCUGGAAAUAAGCUAAUGGAAGAGCAGCUGCAGCAAAGAUGUCAAGACUUGCCAUCAAAGUGAGAGGACCACCUAGUGUCAGCAUGAUCCUAGC

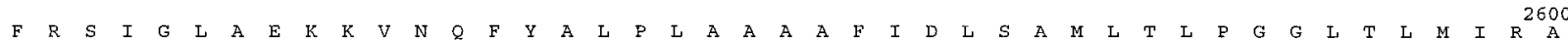
UGUAGUUGCAUGAAACUUGCUACUUGGUUUCAAACCAUAUGCACUUACCAGAGGAAGCCCGCAGAUUUUUUCAUACAUUUUCUGCUUUUCCUUUUCAUCCUUUGUUUCAAUCAGUUCUACGAGCAUUCUA

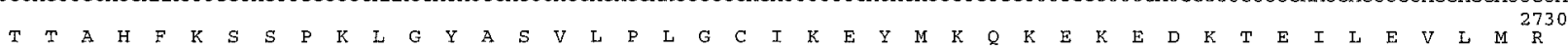
GCUCUGAUAAAGCCUUCCAACCUCCUGAAUGUCCAAUCGUCUUGAUUGGGACUCACAUUAGCACUAGGAAUAACAAUGGUCUUUCCUUGGAAUGUGAAUUUCCCUGUUUUUUUUUGUCUUGUAAAUGCCUG $\begin{array}{lllllllllllllllllllllllllllllllllllllllllllllllllllll}\mathrm{A} & \mathrm{R} & \mathrm{I} & \mathrm{F} & \mathrm{G} & \mathrm{E} & \mathrm{L} & \mathrm{R} & \mathrm{R} & \mathrm{F} & \mathrm{T} & \mathrm{W} & \mathrm{D} & \mathrm{D} & \mathrm{Q} & \mathrm{N} & \mathrm{P} & \mathrm{S} & \mathrm{V} & \mathrm{N} & \mathrm{A} & \mathrm{S} & \mathrm{P} & \mathrm{I} & \mathrm{V} & \mathrm{I} & \mathrm{T} & \mathrm{K} & \mathrm{G} & \mathrm{Q} & \mathrm{F} & \mathrm{T} & \mathrm{F} & \mathrm{K} & \mathrm{G} & \mathrm{T} & \mathrm{K} & \mathrm{I} & \mathrm{T} & \mathrm{K} & \mathrm{Y} & \mathrm{I} & \mathrm{G} & \mathrm{A}\end{array}$ CUCUGUUUCUAAGAAUAGUGUAGCCAUUAUUGAAUGUCAUUUGGACACCUUCAUUUGCCAACACAAAAUUCUUGAAAUUGAAUCCUUCAGUCUCAUCAGAUUCAAUUACCACAUCCACAUCUCCGCCAGA

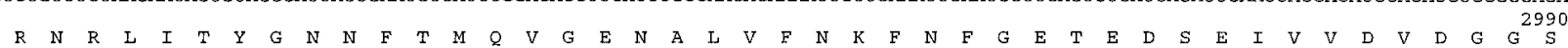

CAGAAGUUUCUCGAUUUCUGACGGUUUCACCCUAACGGUAGACAUGGUGAAUACUUAGGAAGCAGUUUACUCUGAAGAAGUUGUUUGAAUGUUUGUUUUAUACCUCGAJUGCUCU

$\begin{array}{lllllllllllllllllll}\mathrm{L} & \mathrm{L} & \mathrm{K} & \mathrm{E} & \mathrm{I} & \mathrm{E} & \mathrm{S} & \mathrm{P} & \mathrm{K} & \mathrm{V} & \mathrm{R} & \mathrm{V} & \mathrm{T} & \mathrm{S} & \mathrm{M}\end{array}$

3105

Fig. 6. The complete nucleotide sequence of iris yellow spot virus S RNA (numbered from the $5^{\prime}$ end of the viral RNA strand) and the predicted gene products. The deduced amino acid sequence of the protein encoded by the viral RNA is shown above the RNA sequence. The sequence of the protein encoded by the viral complementary strand is shown below the RNA sequence. The asterisk (*) indicates termination codons. 
and PBNV. First, the number of nucleotides (70) of the $5^{\prime}$ nontranslated regions of the IYSV $\mathrm{NS}_{\mathrm{S}}$ and $\mathrm{N}$ genes is exactly the same as in the analogous noncoding regions in PBNV and WSMV. Second, the IYSV N protein (273 aa) more closely resembles those of the Asian isolates in size: WSMV (275 aa) and PBNV (276 aa) rather than the nucleoproteins of TSWV (258 aa), INSV (262 aa), TCSV (259 aa), and GRSV (259 aa).

Whereas sequence alignments of the $\mathrm{N}$ proteins show the lowest identities of IYSV N protein to that of INSV of 30\% (Table 1), the divergence of $\mathrm{NS}_{\mathrm{S}}$ sequences is greater, with identities ranging between 20 and 49\%. A highly conserved stretch of 12 amino acids, located at aa position 400 to 421 of IYSV NS$S_{S}$, was present in all tospoviruses (data not shown). The greater divergence between $\mathrm{NS}_{\mathrm{S}}$ sequences indicates less selective pressure on this protein, suggesting that it is not involved in a basic process during the viral multiplication cycle like genome replication or transcription. Rather, $\mathrm{NS}_{\mathrm{S}}$ may be involved in host range and pathogenesis, because it often forms fibrillar structures (18) as also reported for Rift Valley fever virus $\mathrm{NS}_{\mathrm{S}}$ $(28,29)$, which likewise appears to be involved in pathogenesis.

With respect to the ecological niche of IYSV among the tospoviruses, no firm conclusions can be drawn. The virus apparently has a very narrow experimental host range, but it can be that its natural host range includes monocotyledons other than iris (e.g., onion or lily species). In this respect, it is worthwhile mentioning that, in time and space, onions or leek were cultivated prior or next to the iris cultivation that was found diseased. Sometimes,

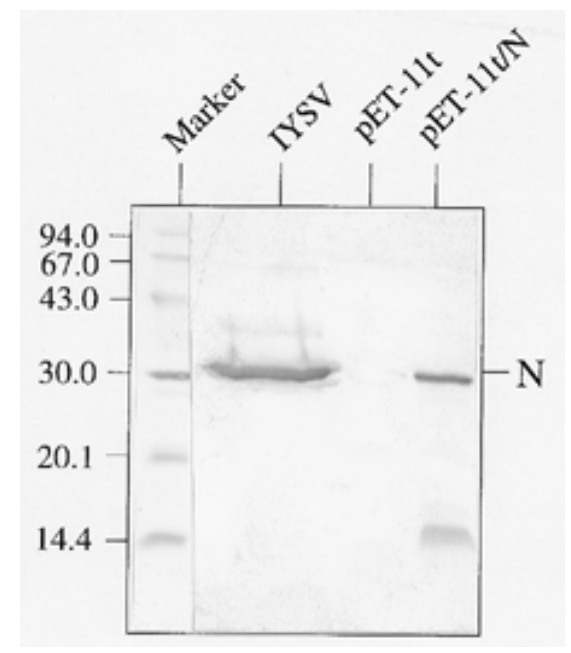

Fig. 7. Expression of iris yellow spot virus (IYSV) nucleoprotein in Escherichia coli. Production of $\mathrm{N}$ protein in E. coli was induced with isopropyl- $\beta$ D-thiogalactopyranoside (IPTG) and the total protein extract was resolved on a 15\% sodium dodecyl sulfate polyacrylamide gel before transferring to a polyvinylidene-difluoride (PVDF) membrane. The western blot was analyzed with $1 \mu \mathrm{g}$ of an IYSV polyclonal anti-N serum per ml. Purified IYSV nucleocapsid material and pET-11t-transformed BL-21 cells were included as controls. Low molecular weight size markers (Amersham Pharmacia Biotech, Uppsala, Sweden) are indicated on the left. symptoms were even observed on leek, and these were initially attributed to a fungus. Meanwhile, IYSV has been found in greenhouse cultivations of leek, as was confirmed by ELISA.

I. hollandica has been cultivated for many years in the Netherlands, and it seems, therefore, unlikely that the virus was introduced with this host. The phylogenetic tree of N-protein sequences (Fig. 8) indicates that IYSV is most closely related to the Asian tospovirus species (WSMV and PBNV). It is, therefore, possible that IYSV has its most important niche in Asia. Possibly, the vector specificity of IYSV can tell more about the origin of this virus, which has only incidentally been found so far and, moreover, in a country where thrips vectoring tospoviruses mainly colonize greenhouse-grown crops and ornamentals.

In conclusion, data presented in this paper support the classification of IYSV as a new tospovirus species, clearly distinct from all other tospovirus species analyzed thus far. Consequently, we propose the establishment of a new serogroup.

\section{ACKNOWLEDGMENTS}

The sequence presented in this paper is accessible in GenBank under number AF001387. This work was supported, in part, by a grant from Program PRAXIS XXI (BD/4560/94), Portugal. We thank R. Goldbach and A.-M. Pereira for their help and suggestions during the preparation of this manuscript; and T. van Kampen (Department of Molecular Biology, Wageningen Agricultural University, the Netherlands) and H. Ramshaw (DNA Sequencing Laboratory, Queen's University at Kingston, Canada) for their help in sequencing.

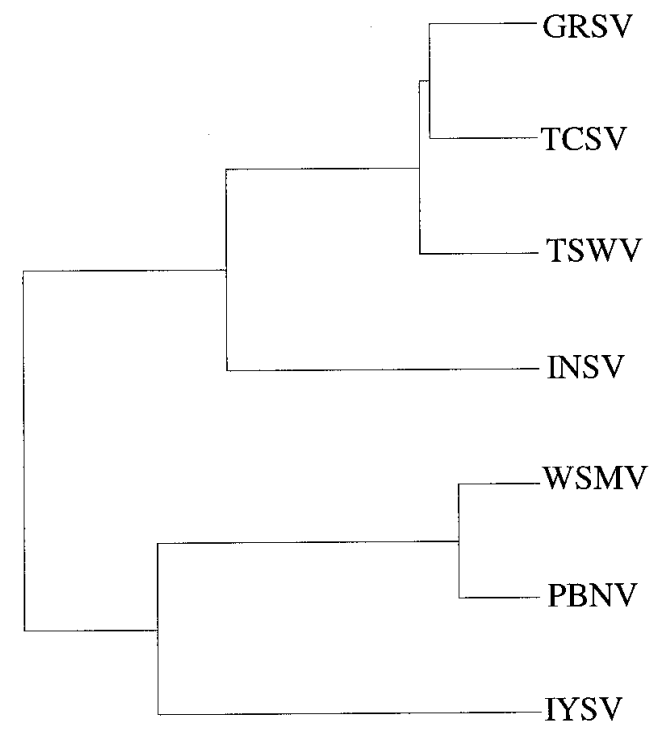

Fig. 8. Phylogenetic tree showing the relationship of iris yellow spot virus to the other representative species within the genus Tospovirus, based on the amino acid sequence of the $\mathrm{N}$ protein. The tree was constructed using Pileup (gap creation penalty $=12$; gap extension penalty $=4$ ) of the GCG package.

TABLE 1. Amino acid sequence identities $(\%)$ of the $\mathrm{N}$ gene in tospoviruses ${ }^{\mathrm{a}}$

\begin{tabular}{|c|c|c|c|c|c|c|c|}
\hline & \multirow{2}{*}{$\frac{\text { Serogroup I }}{\text { TSWV }}$} & \multicolumn{2}{|c|}{ Serogroup II } & \multirow{2}{*}{$\frac{\text { Serogroup III }}{\text { INSV }}$} & \multicolumn{2}{|c|}{ Serogroup IV } & \multirow[b]{2}{*}{ IYSV } \\
\hline & & GRSV & TCSV & & PBNV & WSMV & \\
\hline TSWV & 100 & 78 & 77 & 55 & 33 & 33 & 34 \\
\hline GRSV & $\ldots$ & 100 & 81 & 54 & 34 & 33 & 33 \\
\hline INSV & $\ldots$ & $\ldots$ & $\ldots$ & 100 & 30 & 30 & 30 \\
\hline PBNV & $\ldots$ & $\ldots$ & $\ldots$ & $\ldots$ & 100 & 85 & 44 \\
\hline WSMV & $\ldots$ & $\ldots$ & $\ldots$ & $\ldots$ & $\ldots$ & 100 & 44 \\
\hline
\end{tabular}

${ }^{a}$ Virus names of the representative species of each serogroup. TSWV = tomato spotted wilt virus; GRSV = groundnut ringspot virus; TCSV = tomato chlorotic spot virus; INSV = impatiens necrotic spot virus; PBNV = peanut bud necrosis virus; WSMV = watermelon silver mottle virus; and IYSV = iris yellow spot virus. The tospovirus species referred are the following: TSWV (9); GRSV and TCSV (4); INSV (8); PBNV (27); WSMV (16); and IYSV (this study). 


\section{LITERATURE CITED}

1. Bailey, J. M., and Davidson, N. 1976. Methylmercury as a reversible denaturating agent for agarose gel electrophoresis. Anal. Biochem. 70:75-85.

2. Clark, M. F., and Adams, A. N. 1977. Characteristics of the microplate method of enzyme linked immunosorbent assay for the detection of plant viruses. J. Gen. Virol. 34:475-483.

3. De Ávila, A. C., de Haan, P., Kitajima, E., Kormelink, R., Resende, R. de O., Goldbach, R., and Peters, D. 1992. Characterization of a distinct isolate of tomato spotted wilt virus (TSWV) from Impatiens sp. in the Netherlands. J. Phytopathol. 134:133-151

4. De Ávila, A. C., de Haan, P., Kormelink, R., Resende, R. de O., Goldbach, R., and Peters, D. 1993. Classification of tospoviruses based on phylogeny of nucleoprotein gene sequences. J. Gen. Virol. 74:153-159.

5. De Ávila, A. C., de Haan, P., Kormelink, R., Resende, R. de O., van Poelwijk, F., Wijkamp, I., Goldbach, R., and Peters, D. 1994. Tomato spotted wilt virus: Genome structure, classification, detection and genetically engineered resistance. Pages 95-106 in: Virology in the Tropics. N. Rishi, K. L. Ahuja, and B. P. Singh, eds. Malhotra Publishing House, New Delhi.

6. De Ávila, A. C., de Haan, P., Smeets, M. L. L., Resende, R. de O., Kormelink, R., Kitajima, E., Goldbach, R., and Peters, D. 1992b. Distinct levels of relationships between tospovirus isolates. Arch. Virol. 128:211-227.

7. De Ávila, A. C., Huguenot, C., Resende, R. de O., Kitajima, E. W., Goldbach, R. W., and Peters, D. 1990. Serological differentiation of 20 isolates of tomato spotted wilt virus. J. Gen. Virol. 71:2801-2807.

8. De Haan, P., De Ávila, A. C., Kormelink, R., Westerbroek, A., Gielen, J. J. L., Peters, D., and Goldbach, R. 1992. The nucleotide sequence of the S RNA of impatiens necrotic spot virus, a novel tospovirus. FEBS (Fed. Eur. Biochem. Soc.) Lett. 306:27-32.

9. De Haan, P., Wagemakers, L., Goldbach, R., and Peters, D. 1990. The S RNA segment of tomato spotted wilt virus has an ambisense character. J. Gen. Virol. 71:1001-1007.

10. Deveraux, J., Haeberli, P., and Smithies, O. 1984. A comprehensive set of sequence analysis programs for the VAX. Nucleic Acids Res. 12:387-395.

11. Elliot, R. M. 1990. Molecular biology of the Bunyaviridae. J. Gen. Virol. 73:501-522.

12. Francki, R. I. B., Fauquet, C. M., Knudson, D. D., and Brown, F. 1991. Fifth report of the International Committee on Taxonomy of Viruses. Arch. Virol. Suppl. 2:1-450.

13. Ghanekar, A. M., Reddy, D. V. R., Iizuka, N., Amin, P. W., and Gibbson, R. W. 1979. Bud necrosis of groundnut (Arachis hypogaea) in India caused by tomato spotted wilt virus. Ann. Appl. Biol. 93:173-179.

14. Goldbach, R., and Kuo, G. 1996. Introduction. International symposium on tospoviruses and thrips of floral and vegetable crops. Acta Hortic. 431:21-26.

15. Gubler, V. G., and Hoffman, J. 1983. A simple and very efficient method for generating cDNA libraries. Gene 25:263-269.

16. Heinze, C., Maiss, E., Adam, G., and Casper, R. 1995. The complete nucleotide sequence of the S RNA of a new Tospovirus species, representing serogroup IV. Phytopathology 85:683-690.
17. Kormelink, R., de Haan, P., Meurs, C., Peters, D., and Goldbach, R. 1992. The nucleotide sequence of the M RNA segment of tomato spotted wilt virus, a bunyavirus with two ambisense RNA segments. J. Gen. Virol. 73:2795-2804.

18. Kormelink, R., Kitajima, E. W., De Haan, P., Zuidema, D., Peters, D., and Goldbach, R. 1991. The nonstructural protein $\left(\mathrm{NS}_{\mathrm{S}}\right)$ encoded by the ambisense S RNA segment of tomato spotted wilt virus is associated with fibrous structures in infected plant cells. Virology 181:459-468.

19. Kormelink, R., Storms, M., van Lent, J., Peters, D., and Goldbach, R. W. 1994. Expression and subcellular localization of the $\mathrm{NS}_{\mathrm{M}}$ protein of tomato spotted wilt virus (TSWV), a putative viral movement protein. Virology 200:56-65.

20. Laemmli, U. K. 1970. Cleavage of structural proteins during the assembly of the head of bacteriophage T4. Nature (Lond.) 227:680-685.

21. Law, M. D., and Moyer, J. W. 1990. A tomato spotted wilt-like virus with serologically distinct N protein. J. Gen. Virol. 71:933-938.

22. Law, M. D., Speck, J., and Moyer, J. W. 1991. Nucleotide sequence of the $3^{\prime}$ non-coding region and the $\mathrm{N}$ gene of the S RNA of a serologically distinct tospovirus. J. Gen. Virol. 72:2579-2601.

23. Maiss, E., Ivanova, L., Breyel, E., and Adam, G. 1991. Cloning and sequencing of the S RNA from a Bulgarian isolate of tomato spotted wilt virus. J. Gen. Virol. 72:2597-2601.

24. Maniatis, T., Fritsch, E. F., and Sambrook, K. 1982. Molecular Cloning: A Laboratory Manual. Cold Springs Harbor Laboratory, Cold Springs Harbor, NY.

25. Reddy, D. V. R., Ratna, A. S., Sudarshana, M. R., Poul, F., and Kumar, I. K. 1992. Serological relationships and purification of bud necrosis virus, a tospovirus occurring in peanut (Arachis hypogaea L.) in India. Ann. Appl. Biol. 120:279-286.

26. Sangler, F., Nicklen, S., and Coulson, A. R. 1977. DNA sequencing with chain-terminating inhibitors. Proc. Natl. Acad. Sci. U.S.A. 74:5463-5467.

27. Satyanarayana, T., Mitchell, S. E., Reddy, D. V. R., Brown, S., Kresovich, S., Jarret, R., Naidu, R. A., and Demski, J. W. 1996. Peanut bud necrosis tospovirus S RNA: Complete nucleotide sequence, genome organization and homology to other tospoviruses. Arch. Virol. 141:85-98.

28. Struthers, J. K., and Swanepoel, R. 1982. Identification of a major nonstructural protein in the nuclei of Rift Valley fever virus-infected cells. J. Gen. Virol. 60:381-384.

29. Swanepoel, R., and Blackburn, N. K. 1977. Demonstration of nuclear immunofluorescence in Rift Valley fever infected cells. J. Gen. Virol. 34:557-561.

30. van Kammen, A., Henstra, S., and Ie, T. S. 1966. Morphology of tomato spotted wilt virus. Virology 30:574-577.

31. Vaira, A. M., Roggero, P., Luisoni, E., Masenga, V., Milne, R. G., and Lisa, V. 1993. Characterization of 2 tospoviruses in Italy-Tomato spotted wilt and impatiens necrotic spot. Plant Pathol. 42:530-542.

32. Yeh, S.-D., and Chang, T. F. 1995. Nucleotide sequence of the $\mathrm{N}$ gene of watermelon silver mottle virus, a proposed new member of the genus tospovirus. Phytopathology 85:58-64.

33. Yeh, S.-D., Lin, Y.-C., Cheng, Y. H., Jih, C.-L., Chen, M.-L., and Chen, C-C. 1992. Identification of tomato spotted wilt-like virus infecting watermelon in Taiwan. Plant Dis. 76:835-840. 